Journal of Computer Science 3 (9): 693-699, 2007

ISSN 1549-3636

(C) 2007 Science Publications

\title{
Adaptive Control of Networked Systems in the Presence of Bounded Disturbances
}

\author{
A. H. Tahoun and Fang Hua-Jing \\ School of Information Technology and Engineering, \\ Huazhong University of Science and Technology, Wuhan 430074, China
}

\begin{abstract}
The insertion of data network in the feedback adaptive control loops makes the analysis and design of networked control systems more complex than traditional control systems. This paper addresses the adaptive stabilization problem of linear time-invariant networked control systems when the measurements of the plant states are corrupted by bounded disturbances. The case of state feedback is treated in which only an upper bound on the norm of matrix A is needed. The problem is to find an upper bound on the transmission period $h$ that guarantees the stability of the overall adaptive networked control system under an ideal transmission process, i.e. no transmission delay or packet dropout. Rigorous mathematical proofs are established, that relies heavily on Lyapunov's stability criterion and dead-zone Technique. Simulation results are given to illustrate the efficacy of our design approach.
\end{abstract}

Keywords: Networked control systems, Transmission period, Dead-zone, Lyapunov's stability

\section{INTRODUCTION}

In recent years, the discipline of networked control systems has become a highly active research field. An NCS is a control system in which a data network is used as feedback media. The use of networks as media to interconnect the different components in an industrial control system is rapidly increasing. For example in large scale plants and in geographically distributed systems, where the number and/or location of different subsystems to control make the use of single wires to interconnect the control system prohibitively expensive $^{[1]}$. The primary advantages of an NCS are reduced system wiring, ease of system diagnosis and maintenance, and increase system agility ${ }^{[2]}$.

The insertion of the data network in the feedback control loop makes the analysis and design of an NCS more and more complex, especially for adaptive systems in which systems parameters not completely known. Conventional control theories with many ideal assumptions, such as synchronized control and nondelayed sensing and actuation, must be reevaluated before they can be applied to NCSs. Specifically; the following issues need to be addressed. The first issue is the network induced delay (sensor-to-controller delay and controller-to-actuator delay) that occurs while exchanging data among devices connected to the shared medium. This delay, either constant (up to jitter) or time varying, can degrade the performance of control systems designed without considering the delay and can even destabilize the system. Next, the network can be viewed as a web of unreliable transmission paths. Some packets not only suffer transmission delay but, even worse, can be lost during transmission ${ }^{[3]}$.

The main challenge to be addressed when considering a networked control system is the stability of the overall NCSs. The stability analysis of NCSs is treated in ${ }^{[2-5]}$, with completely known systems. The case of model-based NCS is studied in $^{[1]}$ when the controller/actuator is updated with the sensor information at nonconstant time intervals but with completely known system parameters. A collection of results to determine the closed-loop stability of NCSs in the presence of network sampling, delays, and packet dropouts are covered in ${ }^{[6,7]}$ and references therein, but also for completely known systems. Many of the results presented rely on Lyapunov based techniques and only provide sufficient conditions for stability of the NCS. All these results deal with completely known systems. The disturbance free case of adaptive control of networked systems with unknown plant parameters is first treated in ${ }^{[8,9]}$.

Due to the disturbance, it is no longer possible in general to ensure that the plant states will tend to zero asymptotically with time. An important, if modest, requirement is that the adaptive controller assures the 
uniform boundedness of all the signals in the adaptive loop. The dead-zone idea can be used to prevent the instability by switching off the adaptation algorithm when the resulting error is smaller than a certain threshold.

In this paper, we treat the stability analysis of networked control adaptive systems in the presence of bounded disturbance. Under an ideal transmission process, i.e. no transmission delay or packet dropout, we have derived a sufficient condition on the transmission period that guarantees the NCS will be stable in the presence of bounded disturbamces.

\section{FORMULATION OF THE PROBLEM}

Consider an NCS shown in Fig. 1, in which sensor is clock-driven and both controller and actuator are event driven.

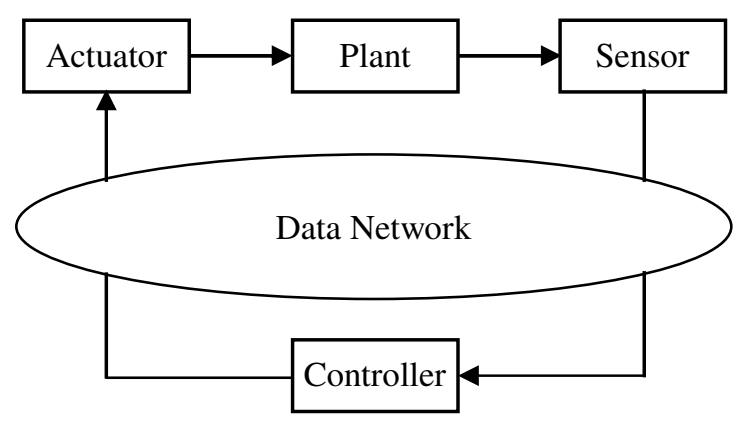

Fig. 1 The block diagram of NCS

In Fig. 1, a class of linear time-invariant plants is described as

$$
\begin{aligned}
\dot{x}(t)=A x(t) & +b u\left(t_{k}\right)+d(t) \\
t & \in\left[t_{k}, t_{k+1}\right), k=0,1,2, \ldots
\end{aligned}
$$

where $x(t) \in R^{\mathrm{n}}$ is a state vector, $u\left(t_{\mathrm{k}}\right) \in R$ is a control input vector, $(A, b)$ is controllable, $A$ is a constant matrix with unknown elements, and $b$ is a known constant vector. In Eq. (1), $d(t)$ is a bounded disturbance vector; that is $\|d(t)\| \leq \rho^{*}$, and $\rho^{*}$ is a known upper bound on the disturbance.

We assume that the control is updated at the instant $t_{k}$ and kept constant until next control update is received at time $t_{k+1}$. Let $h$ be the transmission period between successive transmissions, that is, $h=t_{k+1}-t_{k}$. For this paper, we assume that the transmission process is ideal, there are no delays, no data loss (packet loss) during the transmission. In future work, we will relax these assumptions.

Our objective is to design an adaptive stabilizer for the networked system in the presence of bounded disturbance, and to find an upper bound on the time transmission period (sampling period) $\mathrm{h}$ such that the NCS is still stable.

The control input is of the form

$$
u(t)=k^{T}(t) x\left(t_{k}\right)
$$

where $k(t)$ is an $n$-dimensional control parameter vector, $T$ denotes transpose. From Eqs. (1) and (2), we get

$$
\begin{aligned}
\dot{x}(t) & =A x(t)+b k^{T}\left(t_{k}\right) x\left(t_{k}\right)+d(t) \\
& =\bar{A} x(t)-b k^{* T} x(t)+b k^{T}\left(t_{k}\right) x\left(t_{k}\right)+d(t)
\end{aligned}
$$

Where $\bar{A}=A+b k^{* T}$ is Hurwitz matrix satisfying that $\bar{A}^{T} P+P \bar{A}=-Q, P$ and $Q$ are symmetric and positive-definite matrices, and $k^{*}$ is the true value of $k(t)$. Define $\phi(t)=k(t)-k^{*}$ as the control parameter error vector, and $e(t)=x(t)-x\left(t_{k}\right)$ as the transmission error, Eq. (3) can be rewritten as

$$
\dot{x}(t)=\bar{A} x(t)+b \phi^{T}\left(t_{k}\right) x\left(t_{k}\right)-b k^{* T} e(t)+d(t)
$$

Now, we will turn our attention to the proposed adaptive law and to prove the stability of the overall networked adaptive system.

\section{MAIN RESULT}

To prove the stability of the NCS, firstly, we will find an upper bound on the transmission error $e(t)$, a lower and an upper bound on the state $x(t)$, and finally, we will use these bounds in Lyapunov function to find a bound on the transmission period $h$ to guarantee stability of the overall NCS.

Lemma 1: (Upper bound on the transmission error) The transmission error $\mathrm{e}(\mathrm{t})$ is bounded between two successive transmissions by

$$
\|e(t)\| \leq \gamma\left\|x\left(t_{k}\right)\right\|
$$

where $\gamma=\frac{A_{\text {upp }}+\left\|b k^{T}\left(t_{k}\right)\right\|+\rho^{*}}{A_{u p p}}\left(e^{A_{u p p}\left(t-t_{k}\right)}-1\right), A_{\text {upp }}$ is an 
upper bound on A such that; $\|A\| \leq A_{\text {upp }}$.

Proof: From the definition of $e(t)$, it can be found that

$$
\dot{e}(t)=\left\{\begin{array}{cc}
0 & t=t_{k} \\
\dot{x}(t) & t \in\left[t_{k}^{+}, t_{k+1}\right)
\end{array}\right.
$$

where $t_{k}^{+}$is the time just after $t=t_{k}$. For simplicity we write $t_{k}^{+}$as $t_{k}$. Then, for $t \in\left[t_{k}, t_{k+1}\right)$, we have

$$
\begin{aligned}
\dot{e}(t)=\dot{x}(t)= & A x(t)+b k^{T}\left(t_{k}\right) x\left(t_{k}\right)+d(t) \\
& =A e(t)+A x\left(t_{k}\right)+b k^{T}\left(t_{k}\right) x\left(t_{k}\right)+d(t)
\end{aligned}
$$

Taking the integral on both sides, and taking into account that $e\left(t_{k}\right)=0$, we have

$$
e(t)=e\left(t_{k}\right)+\int_{t_{k}}^{t}\left(A e(s)+A x\left(t_{k}\right)+b k^{T}\left(t_{k}\right) x\left(t_{k}\right)+d(t)\right) d s
$$

Using the assumption that $\|d(t)\| \leq \rho^{*}$, we have

$$
\begin{aligned}
\|e(t)\| \leq & {\left[\|A\|\left\|x\left(t_{k}\right)\right\|+\left\|b k^{T}\left(t_{k}\right)\right\|\left\|x\left(t_{k}\right)\right\|+\rho^{*}\right]\left(t-t_{k}\right) } \\
& +\int_{t_{k}}^{t}\|A\|\|e(s)\| d s
\end{aligned}
$$

If we know an upper bound of $A$ that is; $\|A\| \leq A_{\text {upp }}$, and applying Bellman-Gronwall Lemma ${ }^{[2]}$, yields

$$
\|e(t)\| \leq \int_{t_{k}}^{t}\left[A_{u p p}+\left\|b k^{T}\left(t_{k}\right)\right\|+\rho^{*}\right]\left\|x\left(t_{k}\right)\right\| \exp \left(\int_{s}^{t} A_{u p p} d w\right) d s
$$

Defining

$$
\gamma=\frac{A_{u p p}+\left\|b k^{T}\left(t_{k}\right)\right\|+\rho^{*}}{A_{u p p}}\left(e^{A_{u p p}\left(t-t_{k}\right)}-1\right)
$$

Then, it can be concluded that

$$
\|e(t)\| \leq \gamma\left\|x\left(t_{k}\right)\right\|
$$

This completes the proof of Lemma 1.
Lemma 2: (Upper and lower bounds on NCS states) The state of the NCS, $x(t)$, between successive transmissions is bounded by

$$
(1-\gamma)\left\|x\left(t_{k}\right)\right\| \leq\|x(t)\| \leq(1+\gamma)\left\|x\left(t_{k}\right)\right\|
$$

Proof: As $e(t)=x(t)-x\left(t_{k}\right)$, then

$$
\left\|x\left(t_{k}\right)\right\|-\|e(t)\| \leq\|x(t)\| \leq\|e(t)\|+\left\|x\left(t_{k}\right)\right\|
$$

Using Eq. (5), it can be concluded that

$$
(1-\gamma)\left\|x\left(t_{k}\right)\right\| \leq\|x(t)\| \leq(1+\gamma)\left\|x\left(t_{k}\right)\right\|
$$

This completes the proof of Lemma 2. The main result of this paper will be treated in the following theorem.

Theorem 1: Let an NCS with linear time-invariant plant (1), an adaptive stabilizer with control input (2) is globally stable if the adaptive control law in $^{[10]}$ is modified to take the form

$$
\dot{\phi}(t)=\left\{\begin{array}{lll}
-\alpha x\left(t_{k}\right) x^{T}\left(t_{k}\right) P b & \text { if } & \left\|x\left(t_{k}\right)\right\|>E_{0} \\
0 & \text { if } & \left\|x\left(t_{k}\right)\right\| \leq E_{0}
\end{array}\right.
$$

and the transmission period satisfies

$$
h<\min \left\{h_{1}, h_{2}, h_{3}\right\}
$$

where $E_{0}=\frac{2\|P\| \rho^{*}}{\delta}$, is the size of the dead-zone, $\delta=(1-\gamma)^{2}+\beta-\frac{2 \beta}{3} \gamma, \alpha$ is an $n \times n$ symmetric positivedefinite adaptation gain matrix, and

$$
\begin{gathered}
h_{1}=\frac{1}{A_{u p p}} \ln \left(1+\frac{A_{u p p}}{A_{u p p}+\left\|b k^{T}\left(t_{k}\right)\right\|+\rho^{*}}\right) \\
h_{2}=\frac{1}{A_{u p p}} \ln \left(1+\frac{\beta \lambda_{\min }(Q) A_{u p p}}{\zeta}\right) \\
h_{3}=\frac{1}{A_{u p p}} \ln \left(1+\frac{\left(\left(1-\frac{\beta}{3}\right)-\sqrt{\left(1-\frac{\beta}{3}\right)^{2}-(1-\beta)}\right) A_{u p p}}{A_{u p p}+\left\|b k^{T}\left(t_{k}\right)\right\|+\rho^{*}}\right)
\end{gathered}
$$


where

$$
\begin{gathered}
\zeta=6\|P\|\left(A_{\text {upp }}+\left\|b k^{T}\left(t_{k}\right)\right\|+\rho^{*}\right)\left(1+\lambda_{\min }(Q)+\|\bar{A}\|+A_{\text {upp }}\right. \\
\left.+\|\alpha\|\|P\|\|b\|^{2}\left\|x\left(t_{k}\right)\right\|^{2}+\left\|b k^{T}(t)\right\|\right) .
\end{gathered}
$$

Proof: Consider a positive-definite Lyapunov function $V(t)$ of the form

$$
V(t)=x^{T}(t) P x(t)+\phi^{T}(t) \alpha^{-1} \phi(t)
$$

Differentiating $V(t)$ with respect to $t$, we have

$$
\begin{aligned}
\dot{V}(t)= & \dot{x}^{T}(t) P x(t)+x^{T}(t) P \dot{x}(t) \\
& +\dot{\phi}^{T}(t) \alpha^{-1} \phi(t)+\phi^{T}(t) \alpha^{-1} \dot{\phi}(t)
\end{aligned}
$$

Substituting for $\dot{x}(t)$ and $\dot{\phi}(t)$ from Eqs. (4) and (7), there results

$$
\begin{aligned}
\dot{V}(t)= & x^{T}(t) \bar{A} P x(t)+x^{T}\left(t_{k}\right) \phi\left(t_{k}\right) b^{T} P x(t) \\
& -e^{T}(t) k^{*} b^{T} P x(t)+x^{T}(t) P \bar{A} x(t) \\
& +x^{T}(t) P b \phi^{T}\left(t_{k}\right) x\left(t_{k}\right) \\
& -x^{T}(t) P b k^{* T} e(t) \\
& -b^{T} P x\left(t_{k}\right) x^{T}\left(t_{k}\right) \phi(t) \\
& -\phi^{T}(t) x\left(t_{k}\right) x^{T}\left(t_{k}\right) P b \\
& +x^{T}(t) P d(t)+d^{T}(t) P x(t)
\end{aligned}
$$

Rearranging Eq. (10), yields

$$
\begin{aligned}
\dot{V}(t)= & -x^{T}(t) Q x(t) \\
& +2 x^{T}(t) P b \phi^{T}\left(t_{k}\right) x\left(t_{k}\right) \\
& -2 x^{T}(t) P b k^{* T} e(t) \\
& -2 x^{T}\left(t_{k}\right) P b \phi^{T}(t) x\left(t_{k}\right) \\
& +2 x^{T}(t) P b \phi^{T}(t) x\left(t_{k}\right) \\
& -2 x^{T}(t) P b \phi^{T}(t) x\left(t_{k}\right) \\
& +2 x^{T}(t) P d(t)
\end{aligned}
$$

$\dot{V}(t)$ becomes bounded from above as

$$
\begin{aligned}
\dot{V}(t) \leq & -\lambda_{\min }(Q)\|x(t)\|^{2} \\
& +2\|P\|\left\|b \phi^{T}(t)\right\|\|e(t)\|\left\|x\left(t_{k}\right)\right\| \\
& +2\|P\|\left\|b k^{* T}\right\|\|x(t)\|\|e(t)\| \\
& +2\|P\|\left\|b k^{T}\left(t_{k}\right)-b k^{T}(t)\right\|\|x(t)\|\left\|x\left(t_{k}\right)\right\| \\
& +2\|P\|\|x(t)\| \rho^{*}
\end{aligned}
$$

From (5) and (6), then

$$
\begin{aligned}
\dot{V}(t) \leq & -\lambda_{\text {min }}(Q)\|x(t)\|^{2} \\
& +\frac{2 \gamma}{(1-\gamma)}\|P\|\left\|b \phi^{T}(t)\right\|\|x(t)\|\left\|x\left(t_{k}\right)\right\| \\
& +2 \gamma\|P\|\left\|b k^{* T}\right\|\|x(t)\|\left\|x\left(t_{k}\right)\right\| \\
& +2\|P\|\left\|b k^{T}\left(t_{k}\right)-b k^{T}(t)\right\|\|x(t)\|\left\|x\left(t_{k}\right)\right\| \\
& +2\|P\|\|x(t)\| \rho^{*}
\end{aligned}
$$

Using (6), and rearranging,

$$
\begin{aligned}
\dot{V}(t) \leq \frac{1}{(1-\gamma)}\|x(t)\| & {\left[\| x ( t _ { k } ) \| \left(-(1-\gamma)^{2} \lambda_{\min }(Q)\right.\right.} \\
& +2 \gamma\|P\|\left\|b k^{T}(t)-b k^{* T}\right\| \\
& +2 \gamma(1-\gamma)\|P\|\left\|b k^{* T}\right\| \\
& \left.+2(1-\gamma)\|P\|\left\|b k^{T}\left(t_{k}\right)-b k^{T}(t)\right\|\right) \\
& \left.+2\|P\| \rho^{*}\right]
\end{aligned}
$$

By choosing $\gamma<1$, we have $h<h_{l}$, where

$$
h_{1}=\frac{1}{A_{u p p}} \ln \left(1+\frac{A_{u p p}}{A_{u p p}+\left\|b k^{T}\left(t_{k}\right)\right\|+\rho^{*}}\right)
$$

From Eq. (7), it can be found that

$$
\left\|b k^{T}(t)-b k^{T}\left(t_{k}\right)\right\| \leq\|\alpha\|\|b\|^{2}\left\|x\left(t_{k}\right)\right\|^{2}\|P\| h
$$

Then $\dot{V}(t)$ becomes 


$$
\begin{aligned}
\dot{V}(t) \leq \frac{1}{(1-\gamma)} & \|x(t)\|\left[\| x ( t _ { k } ) \| \left(-(1-\gamma)^{2} \lambda_{\text {min }}(Q)\right.\right. \\
& +2 \gamma\|P\|\left\{\left\|b k^{T}(t)\right\|+\|\bar{A}\|+A_{\text {upp }}\right\} \\
& +2 \gamma(1-\gamma)\|P\|\left\{\|\bar{A}\|+A_{u p p}\right\} \\
& \left.+2 \gamma(1-\gamma)\|P\|^{2}\|\alpha\|\|b\|^{2}\left\|x\left(t_{k}\right)\right\|^{2}\right) \\
& \left.+2\|P\| \rho^{*}\right]
\end{aligned}
$$

Defining

$$
\begin{gathered}
\zeta=6\|P\|\left(A_{\text {upp }}+\left\|b k^{T}\left(t_{k}\right)\right\|+\rho^{*}\right)\left(1+\lambda_{\min }(Q)+\|\bar{A}\|+A_{\text {upp }}\right. \\
\left.+\|\alpha\|\|P\|\|b\|^{2}\left\|x\left(t_{k}\right)\right\|^{2}+\left\|b k^{T}(t)\right\|\right) .
\end{gathered}
$$

and, by choosing $\gamma<\frac{\beta \lambda_{\min }(Q)}{\zeta}, 0<\beta<1$, we have $h<h_{2}$, where

$$
h_{2}=\frac{1}{A_{u p p}} \ln \left(1+\frac{\beta \lambda_{\min }(Q) A_{u p p}}{\zeta}\right)
$$

Substituting for $\gamma$ in (16), we get

$$
\dot{V}(t) \leq \frac{\lambda_{\min }(Q)}{(1-\gamma)}\|x(t)\|\left[\left(-(1-\gamma)^{2}+\beta-\frac{2 \beta}{3} \gamma\right)\left\|x\left(t_{k}\right)\right\|+2\|P\| \rho^{*}\right]
$$

Finally, by choosing

$$
\gamma<\left(1-\frac{\beta}{3}\right)-\sqrt{\left(1-\frac{\beta}{3}\right)^{2}-(1-\beta)}
$$

we have $h<h_{3}$, where

$$
h_{3}=\frac{1}{A_{u p p}} \ln \left(1+\frac{\left(\left(1-\frac{\beta}{3}\right)-\sqrt{\left(1-\frac{\beta}{3}\right)^{2}-(1-\beta)}\right) A_{u p p}}{A_{u p p}+\left\|b k^{T}\left(t_{k}\right)\right\|+\rho^{*}}\right)
$$

From (18) and (19), it can be found that

$$
\dot{V}(t) \leq \frac{\lambda_{\min }(Q)}{(1-\gamma)}\|x(t)\|\left[-\delta\left\|x\left(t_{k}\right)\right\|+2\|P\| \rho^{*}\right]
$$

where $\delta$ is defined by

$$
\delta=(1-\gamma)^{2}+\beta-\frac{2 \beta}{3} \gamma
$$

By choosing $\gamma$ as in (19), it can be proved that $\delta$ is positive. Then, to guarantee that $\dot{V}(t)<0$, we must have

$$
\left\|x\left(t_{k}\right)\right\|>E_{0}
$$

where $E_{0}=\frac{2\|P\| \rho^{*}}{\delta}$.

So, the adaptation must stop if $\left\|x\left(t_{k}\right)\right\| \leq E_{0}$, this is the idea of the dead-zone technique ${ }^{[10]}$. From the above, we can conclude that, using the adaptive law Eq. (7), $\dot{V}(t)<0$ if $h$ satisfies $h<\min \left\{h_{1}, h_{2}, h_{3}\right\}$ defined in Eqs. (15), (17), and (20), respectively. Therefore, $x(t)$, $\phi(t)$, and $V(t)$ are bounded for all $t \geq t_{0}$ and the over all system is globally stable.

\section{SIMULATION RESULTS}

Now, we demonstrate the applicability of our approach through the following example. Consider the plant and the desired plant parameters

$$
A=\left[\begin{array}{ll}
0 & 2 \\
1 & 0
\end{array}\right], \quad B=\left[\begin{array}{l}
1 \\
1
\end{array}\right], \quad \bar{A}=\left[\begin{array}{cc}
-1 & 0 \\
0 & -2
\end{array}\right]
$$

Let

$$
P=\left[\begin{array}{ll}
1 & 0 \\
0 & 1
\end{array}\right], \quad Q=\left[\begin{array}{ll}
2 & 0 \\
0 & 4
\end{array}\right]
$$

Also, let the disturbance vector

$$
d(t)=\left[\begin{array}{l}
0.1 \sin 5 t \\
0.1 \sin 5 t
\end{array}\right]
$$

with $A$ is unknown but only $A_{\text {upp }}$ is known (take $A_{\text {upp }}=$ 
3). Figure 2 shows the simulation results for the networked control system with $x(0)=\left[\begin{array}{ll}1 & 1\end{array}\right]^{T}, \alpha=I$ (identity matrix), $\beta=0.9, k(0)=\left[\begin{array}{ll}0 & 0\end{array}\right]^{T}$, it is found that $h_{1}<0.2205 \mathrm{~s}, h_{2}<0.0077 \mathrm{~s}$, and $h_{3}<0.0228 \mathrm{~s}$. Before starting simulation we know that, $h<0.0077 \mathrm{~s}$, but with simulation proceeds, $h$ can be found on-line as shown in Fig. 3 ( we take $h=0.002 \mathrm{~s}$ ). Figure 4 shows the simulation results for the networked control system with $x(0)=\left[\begin{array}{ll}1 & 1\end{array}\right]^{T}, \alpha=I, \beta=0.9, k(0)=\left[\begin{array}{ll}1 & 1\end{array}\right]^{T}$, it is found that $h_{1}<0.1518 \mathrm{~s}, h_{2}<0.0040 \mathrm{~s}$, and $h_{3}<$ 0.0142s. Before starting simulation we know that, $h<$ $0.0040 \mathrm{~s}$ (we take $h=0.001 \mathrm{~s}$ ), also with simulation proceeds, $h$ can be found on-line as shown in Fig. 5 .

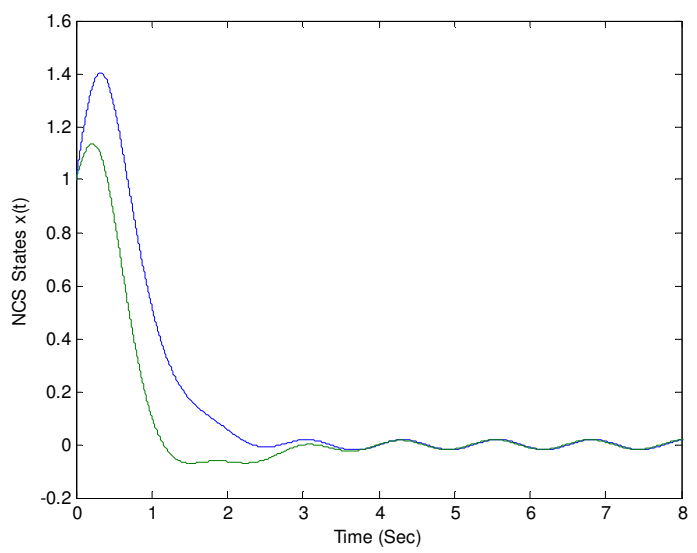

Fig. 2: NCS states $x(t)$

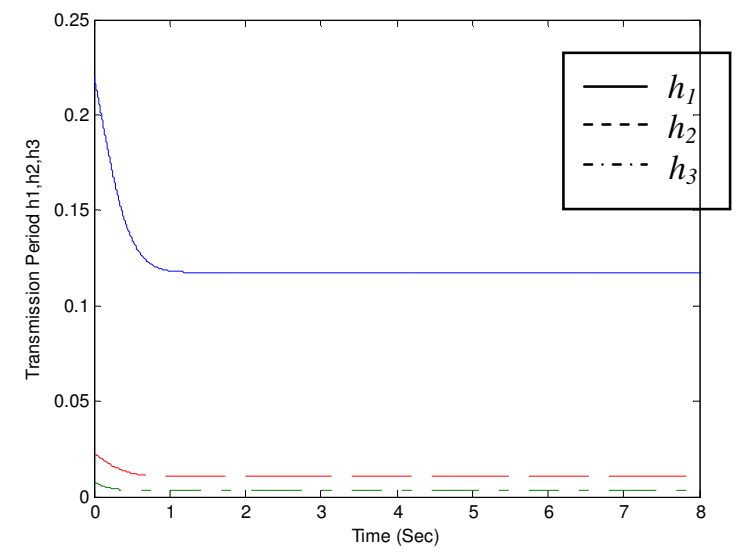

Fig. 3: Transmission period $h$

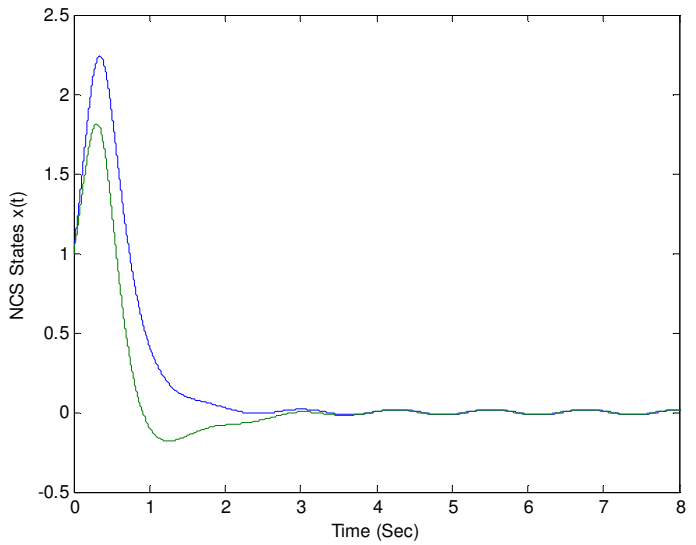

Fig. 4: NCS states $x(t)$

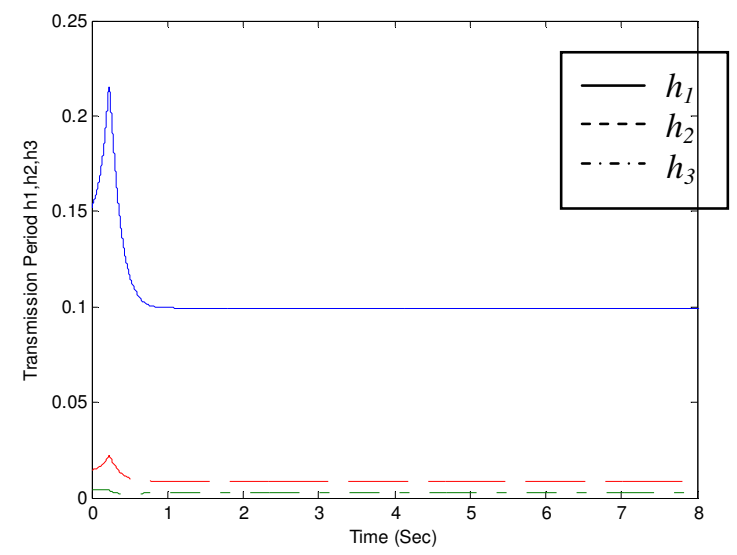

Fig. 5: Transmission period $h$

\section{CONCLUSION}

The paper addresses the adaptive stabilization problem of linear time-invariant networked control systems in the presence of bounded disturbances on the plant states. The case of state feedback is in which only an upper bound on the norm of matrix $A$ is required. As shown in theorem 1, the priori knowledge of upper bound on norm $A$ is not required in constructing the controller but it is required only to determine an upper bound on the transmission period $h$ that guarantees the stability of the overall adaptive networked control system under an ideal transmission process, i.e. no transmission delay or packet dropout. The dead-zone idea is used to prevent the instability by switching off the adaptation algorithm when the resulting error is smaller than a certain threshold .In future work we will try to relax these assumptions. Rigorous mathematical proofs are established relies heavily on Lyapunov's 
stability criterion. Simulation results are given to illustrate the efficacy of our design approach. It is verified that, if the sampling period of the network is less than the upper bound on $h$, the control parameters of the adaptive controller are bounded and that the NCS states converge to zero as time tends to infinity value as time evolves

\section{ACKNOWLEDGEMENTS}

This work is supported by National Natural Science Foundation of China, Grant \#60574088 and \#60274014.

\section{REFERENCES}

1. Montestruque L. A., and P. Antsaklis, 2004. Stability of model-based networked control systems with time-varying transmission times. IEEE Trans. Automat. Contr., vol. 49, no. 9, pp. 1562-1572.

2. Zhang W., 2001. Stability analysis of networked control systems. PhD Thesis, Case Westem Reserve University.

3. Zhang W., M. S. Branicky, and S. M. Phillips, 2001. Stability of networked control systems. IEEE Control System Magzine, vol. 21, pp. 84-99.
4. Walsh G. C., H. Ye, and L. Bushnell, 1999. Stability analysis of networked control systems. in Proc. Amer. Control Conf., San Diego, CA, pp. 2876-2880.

5. Ye H., 2000. Research on networked control systems. PhD Thesis, University of Maryland.

6. Baillieul J. and P. J. Antsaklis. 2007. Control and Communication Challenges in Networked Control Systems. IEEE Control System Magzine, vol. 95, pp. 9-28.

7. Hespanha J. P., P. N. Naghshtabrizi, and Y. Xu. 2007. A Survey of Recent Results in Networked Control Systems. IEEE Control System Magzine, vol. 95, pp. 138-162.

8. Tahoun A. H. and F. Hua-Jing, 2007. A Novel Approach to Adaptive Control of Networked Systems. Ubiquitous Computing and Communication Journal, vol. 2 no. 4.

9. Tahoun A. H. and F. Hua-Jing, In Press. Adaptive Stabilization of Networked Control Systems. Journal of Applied Sciences.

10. Tao G., 2003. Adaptive Control Design and Analysis. John Wiley \& Sons, Inc., New Jersy. 